\title{
CHARACTERIZING RINGS BY A DIRECT DECOMPOSITION PROPERTY OF THEIR MODULES
}

\author{
DINH VAN HUYNH ${ }^{\curvearrowleft}$ and S. TARIQ RIZVI
}

(Received 23 June 2003; revised 27 January 2005)

Communicated by J. Du

\begin{abstract}
A module $M$ is said to satisfy the condition $\left(\wp^{*}\right)$ if $M$ is a direct sum of a projective module and a quasi-continuous module. In an earlier paper, we described the structure of rings over which every (countably generated) right module satisfies $\left(\wp^{*}\right)$, and it was shown that such a ring is right artinian. In this note some additional properties of these rings are obtained. Among other results, we show that a ring over which all right modules satisfy $\left(\wp^{*}\right)$ is also left artinian, but the property $\left(\wp^{*}\right)$ is not left-right symmetric.
\end{abstract}

2000 Mathematics subject classification: primary 16D70, 16P20, 16D50.

\section{Introduction}

Throughout this note, all rings are associative with identity, and all modules are unitary modules. Let $M$ be a right module over a ring $R$. The Jacobson radical and the injective hull of $M$ are denoted respectively by $J(M)$ and $E(M)$. For a module $M$ consider the following conditions:

$\left(\mathrm{C}_{1}\right)$ Every submodule of $M$ is essential in a direct summand of $M$.

$\left(\mathrm{C}_{2}\right)$ Every submodule isomorphic to a direct summand of $M$ is itself a direct summand.

$\left(\mathrm{C}_{3}\right)$ If $A, B$ are direct summands of $M$ with $A \cap B=0$, then $A \oplus B$ is a direct summand of $M$.

A module $M$ is defined to be a $C S$ module (or an extending module) if $M$ satisfies condition $\left(\mathrm{C}_{1}\right)$. If $M$ satisfies $\left(\mathrm{C}_{1}\right)$ and $\left(\mathrm{C}_{2}\right)$, then $M$ is said to be a continuous module. A module $M$ is called quasi-continuous if it satisfies $\left(\mathrm{C}_{1}\right)$ and $\left(\mathrm{C}_{3}\right)$.

(C) 2006 Australian Mathematical Society $1446-7887 / 06 \$ A 2.00+0.00$ 
Let $M$ be a module. A module $N$ is called $M$-injective if every homomorphism of any submodule $L \subseteq M$ to $N$ can be extended to a homomorphism of $M$ to $N$. A module $N$ is called quasi-injective (or self-injective), if $N$ is $N$-injective.

If $M$ is a module of finite composition length, we denote its length by $l(M)$.

Following [5], a module $M$ is said to satisfy the condition $\left(\wp^{*}\right)$ if $M$ is a direct sum of a projective module and a quasi-continuous module. A ring $R$ is called a right $\wp^{*}$-semisimple ring, if every right $R$-module satisfies $\left(\wp^{*}\right)$. Rings whose countably generated right modules satisfy $\left(\wp^{*}\right)$ were characterized in [5, Theorem 7]. These rings are exactly right artinian rings over which every finitely generated right module is a direct sum of a projective module and a quasi-injective module (and in particular, are also right $\wp^{*}$-semisimple). In this note we improve this result by showing:

(1) Every right $\wp^{*}$-semisimple ring is left artinian.

(2) A right $\wp^{*}$-semisimple ring is not necessarily left $\wp^{*}$-semisimple.

(3) In general, the direct sum decomposition of $R$ in [5, Theorem 7 (III)] is not a ring-direct sum decomposition.

(4) Finally we give a correction that the right ideal $B$ of $R$ is not necessarily a CS right $R$-module as claimed in [5, Theorem 7 (III) (ii) and Lemma 11].

Thus, combining with [5, Theorem 7], we describe the structure of right $\wp^{*}$ semisimple rings in the following theorem.

THEOREM 1.1. For a ring $R$, the following conditions are equivalent:

(I) Every countably generated right $R$-module satisfies $\left(\wp^{*}\right)$.

(II) $R$ is right artinian and every finitely generated right $R$-module satisfies ( $\left.\wp^{*}\right)$.

(III) $R$ is a right and left artinian ring with Jacobson radical square zero; $R_{R}=A \oplus B \oplus C$, where $(B \oplus C) A=B C=C B=0$, and $B_{R}$ and $C_{R}$ are nonsingular right ideals of $R$. In general, this direct sum is not a ring-direct sum. Moreover,

(i) $A_{R}=A_{1} \oplus \cdots \oplus A_{l}$, where each $A_{i}$ is uniform, $E\left(A_{i}\right)$ is projective, and $l\left(E\left(A_{i}\right)\right) \leqslant 2$.

(ii) $B_{R}=B_{1} \oplus \cdots \oplus B_{m}$, where each $B_{j}$ is a uniform module of length one or two; the injective hull $E(S)$ of each minimal submodule $S$ of $B_{R}$ has length three. Moreover, $E(S) / S$ is a direct sum of two simple modules, in particular $E(S)=x R+y R$ for some $x, y \in E(S)$. If $B \neq 0$, then there exist at least two (uniform) direct summands $B_{j}$ and $B_{j^{\prime}}$ of $B$ with $l\left(B_{j}\right)=1, l\left(B_{j^{\prime}}\right)=2$ and $B_{j} \cong \operatorname{Soc}\left(B_{j^{\prime}}\right)$. Furthermore, $B_{R}$ is not necessarily $C S$ and has the structure described in Proposition 3.2.

(iii) $C_{R}=C_{1} \oplus \cdots \oplus C_{q}$, where each $C_{k}$ is an indecomposable module of length one or three; the injective hull of each minimal submodule of $C_{R}$ is of length two and not projective. If $C \neq 0$, there exist at least two $C_{k}$, say $C_{1}, C_{2}$ with $l\left(C_{1}\right)=1$, $l\left(C_{2}\right)=3$ and $C_{1}$ is embedable in $\operatorname{Soc}\left(C_{2}\right)$. 
(IV) Every right $R$-module is a direct sum of a projective module and a quasiinjective module. In particular, $R$ is right $\wp^{*}$-semisimple.

In general, right $\wp^{*}$-semisimple rings need not be left $\wp^{*}$-semisimple.

\section{The proof of Theorem 1.1}

We refer to [5, Theorem 7] for the stucture of a right $\wp^{*}$-semisimple ring. Hence, in addition to [5, Theorem 7], for a right $\wp^{*}$-semisimple ring $R$ we need to prove:

(1) $R$ is left artinian.

(2) The direct sum decomposition $R_{R}=A \oplus B \oplus C$ in [5, Theorem 7 (III)] is not necessarily a ring-direct sum decomposition.

(3) $R$ is not necessarily left $\wp^{*}$-semisimple.

(4) In general, $B_{R}$ in (ii) of [5, Theorem 7 (III)] is not CS.

ProOF. (1) By [5, Theorem 7], $R$ is right artinian, and for any right $R$-module $M$, $M=P \oplus Q$, where $P_{R}$ is projective, and $Q_{R}$ is quasi-injective. By [1, Theorem 27.11], $P$ is a direct sum of cyclic modules, each of which is isomorphic to some $e R$ with a primitive idempotent $e^{2}=e \in R$. As $R$ is right artinian, $Q=\oplus_{i \in l} U_{i}$, where each $U_{i}$ is uniform and isomorphic to the quasi-injective hull of some simple right $R$-module (compare with [7]). By [5, Theorem 7], each $E\left(S_{i}\right)$ is 2-generated. But, as a right artinian ring, $R$ has only finitely many non-isomorphic simple right $R$-modules, and finitely many non-isomorphic indecomposable projective right $R$-modules. It follows that $R$ has only finitely many non-isomorphic indecomposable right $R$-modules, or in other words, $R$ is a ring of finite representation type. Thus it is well-known that $R$ is left artinian.

(2) We consider the following example.

Let $\mathbb{C}$ and $\mathbb{R}$ be the fields of complex numbers and real numbers, respectively. Let $V=\left\{\left(\begin{array}{ll}0 & y \\ 0 & 0\end{array}\right) \mid y \in \mathbb{C}\right\} \subset\left\{\left(\begin{array}{ll}x & y \\ 0 & x\end{array}\right) \mid x, y \in \mathbb{C}\right\}, K=\left\{\left(\begin{array}{ll}x & 0 \\ 0 & x\end{array}\right) \mid x \in \mathbb{C}\right\}$, and $F=\left\{\left(\begin{array}{ll}x & 0 \\ 0 & x\end{array}\right) \mid x \in \mathbb{R}\right\}$. Then $V$ is a $K$-bialgebra with $\operatorname{dim}\left(V_{K}\right)=\operatorname{dim}\left({ }_{K} V\right)=1$, $\operatorname{dim}\left(V_{F}\right)=\operatorname{dim}\left({ }_{F} V\right)=2, V^{2}=0$, and $K V=V K=F V=V F=V$. Notice that $K \cong \mathbb{C}, F \cong \mathbb{R}$, and $F$ is a subfield of $K$ with $\operatorname{dim}\left(K_{F}\right)=2$. We consider the ring

$$
R=\left(\begin{array}{ccc}
K & V & 0 \\
0 & K & V \\
0 & 0 & F
\end{array}\right)
$$

and aim to show first that $R$ is a right $\wp^{*}$-semisimple ring.

Matrix rings of this type are very useful in describing the structure of some other interesting classes of rings, see [6]. 
Let

$$
L_{1}=\left(\begin{array}{ccc}
K & 0 & 0 \\
0 & 0 & 0 \\
0 & 0 & 0
\end{array}\right), \quad L_{2}=\left(\begin{array}{ccc}
0 & V & 0 \\
0 & K & 0 \\
0 & 0 & 0
\end{array}\right), \quad L_{3}=\left(\begin{array}{ccc}
0 & 0 & 0 \\
0 & 0 & V \\
0 & 0 & F
\end{array}\right)
$$

Then ${ }_{R} R=L_{1} \oplus L_{2} \oplus L_{3}$, a direct sum of three local left ideals with $l\left(L_{1}\right)=1, l\left(L_{2}\right)=$ $l\left(L_{3}\right)=2$. In particular, $R$ is left serial. Moreover, $R / J(R) \cong K \oplus K \oplus F$, that is, commutative. Hence by [3, Theorem 3.2], the injective hull of every simple right $R$-module is uniserial, that is, its lattice of submodules is linearly ordered by inclusion. Let $S$ be a simple right $R$-module, and let $E(S)$ be the injective hull of $S$. As $J(R)^{2}=0$, we have $E(S) J(R) \subseteq S$. This shows that the uniserial module $E(S) / S$ is semisimple, hence it is zero or simple. Therefore $l(E(S)) \leqslant 2$.

Set

$$
A_{1}=\left(\begin{array}{ccc}
K & V & 0 \\
0 & 0 & 0 \\
0 & 0 & 0
\end{array}\right), \quad C_{1}=\left(\begin{array}{ccc}
0 & 0 & 0 \\
0 & K & V \\
0 & 0 & 0
\end{array}\right), \quad C_{2}=\left(\begin{array}{ccc}
0 & 0 & 0 \\
0 & 0 & 0 \\
0 & 0 & F
\end{array}\right)
$$

Then $A_{1}$ is injective, because $l\left(A_{1}\right)=2$. Moreover, $C_{1}$ and $C_{2}$ are nonsingular right ideals, $C_{1}$ has length 3 , and uniform dimension 2. Each simple submodule of $C_{1}$ is isomorphic to $C_{2}$.

Write $\operatorname{Soc}\left(C_{1}\right)=S \oplus T$ where $S, T$ are minimal right ideals. Let $T^{*}$ be a maximal essential extension of $T$ in $C_{1}$, that is, $T^{*}$ is a closure of $T$ in $C_{1}$. If $l\left(T^{*}\right)>1$, then $T^{*} \oplus S=C_{1}$, a contradiction. Hence $l\left(T^{*}\right)=1$, or equivalently, $T^{*}=T$, that is $T$ is a closed submodule of $C_{1}$. Therefore, $C_{1} / T$ is uniform (compare with [2, Section $5.10(1)]$ ), and it has length 2 . Whence $C_{1} / T$ must be injective, and since $S$ embeds in $C_{1} / T$, we have $E(S) \cong C_{1} / T$. Moreover, $C_{1} / T$ is not projective, because otherwise $T$ would split in $C_{1}$. A similar consideration yields that $C_{1} / S$ is injective, uniform, not projective, $l\left(C_{1} / S\right)=2$ and $E(T) \cong C_{1} / S$. It follows that $E\left(C_{2}\right)$ is also not projective, and $l\left(E\left(C_{2}\right)\right)=2$.

Set $A=A_{1}, C=C_{1} \oplus C_{2}$. Then $R=A \oplus C$ and $C A=0$. Thus $R$ is a ring of Theorem 1.1 with $B=0$, but $A C \neq 0$. This proves (2).

(3) We consider the left side of the above right $\wp^{*}$-semisimple ring $R$. Let $L_{i}$ be as before. It is easy to check that $L_{3}$ is a two-sided ideal of $R$, for which we have

$$
R / L_{3} \cong\left(\begin{array}{cc}
K & V \\
0 & K
\end{array}\right) \cong\left(\begin{array}{ll}
K & K \\
0 & K
\end{array}\right)
$$

It follows that $\left(\begin{array}{ll}0 & V \\ 0 & K\end{array}\right)$ is an injective left ideal of $R / L_{3}$. Hence $\left.\left(L_{2}+L_{3}\right) / L_{3} \cong L_{2}\right)$ is an injective left $R / L_{3}$-module. Therefore $L_{2}$ is a quasi-injective left ideal of $R$. 
We aim to show that it is even an injective left $R$-module. It is obvious that $L_{2}$ is $L_{1}$-injective. Let

$$
T=\left(\begin{array}{ccc}
K & V & 0 \\
0 & 0 & V \\
0 & 0 & 0
\end{array}\right) .
$$

Then $T$ is an essential left ideal of $R$. As $V^{2}=0$, it is clear that $T \operatorname{Soc}\left(L_{3}\right)=0$. This means that $\operatorname{Soc}\left(L_{3}\right)$ is a singular left ideal of $R$. As $L_{2}$ is a nonsingular left ideal of $R$, there is no nonzero map from submodules of $L_{3}$ to $L_{2}$. This shows that $L_{2}$ is $L_{3}$-injective. Thus by [1, Section 16.13 (2)], $L_{2}$ is $\left(L_{1} \oplus L_{2} \oplus L_{3}=R\right.$ )-injective, as claimed.

Now if $R$ is left $\wp^{*}$-semisimple, so applying [5, Theorem 7] for left $\wp^{*}$-semisimple rings we see that $L_{3}$ must be injective. This means that $R$ is a direct sum of a simple left ideal and two injective uniform left ideals of length 2. By [2, Section $13.5(\mathrm{e}),(\mathrm{g})]$, $R$ must be right serial also. However, this is impossible because the local right ideal $C_{1}$ defined in the proof of (2) is not uniform. Thus $R$ is not left $\wp^{*}$-semisimple, completing the proof of (3).

We prove (4) by giving a more general observation on CS modules in the next section. In particular, in Proposition 3.2, we will give more information on the structure of the right ideal $B \subseteq R$ of Theorem 1.1.

\section{A correction}

The conclusion in (ii) of [5, Theorem 7 (III)] and [5, Lemma 11], that $B_{R}$ is CS, is unfortunately incorrect. This mistake arose from an incorrect conclusion in the proof of [5, Theorem 7] on page 144, line 6, that ' $\operatorname{ann}_{R}(w)=\operatorname{ann}_{R}(u r) \cap \operatorname{ann}_{R}(v s)$ if $w=u r+v s^{\prime}$. Fortunately, this mistake does not affect the correctness of other parts of Theorem 1.1, because the CS conclusion for $B_{R}$ was not used anywhere in the remainder of the proof of [5, Theorem 7]. In (a) of the proof of [5, Lemma 13] the fact that $B_{R}$ is a direct sum of uniform modules was used, but this property follows from the definition of $B_{R}$ and not because $B_{R}$ was CS.

For the purpose of showing that the right ideal $B$ of $R$ in [5, Theorem 7] is, in general, not a CS right $R$-module, we first prove a general result, which might also be of interest on its own.

For a module $M_{R}$ over a ring $R$ we denote by $\sigma[M]$ the full subcategory of Mod- $R$ whose objects are submodules of $M$-generated modules. For $N \in \sigma[M]$, the injective hull of $N$ in $\sigma[M]$ is denoted by $E_{M}(N)$. It is known that $E_{M}(N)$ is $M$-injective and for each nonzero proper submodule $T$ of $E_{M}(N), T$ is not $M$-injective. This fact is used in the proof of Lemma 3.1 below. For more on basic properties of $E_{M}(N)$ we refer to $[8$, Section 15$]$. 
LEMMA 3.1. For a right module $M_{R}$ over a ring $R$, let $M_{R}=M_{1} \oplus \cdots \oplus M_{t} \oplus$ $M_{t+1} \oplus \cdots \oplus M_{n}$, such that each $M_{i}$ is uniform, $l\left(M_{1}\right)=\cdots=l\left(M_{t}\right)=2,(t \geqslant 1)$, and $l\left(M_{t+1}\right)=\cdots=l\left(M_{n}\right)=1$. Assume further that $\operatorname{Soc}\left(M_{i}\right) \cong \operatorname{Soc}\left(M_{j}\right)$, and $l\left(E_{M}\left(M_{i}\right)\right)>2$ for all $i, j=1,2, \ldots, n$. Then $M$ is a CS module if and only if $t=1$.

PROOF. Let $t=1$ and let $V$ be a closed submodule of $M$. If $M_{1} \cap V=0$, then by modularity we have $M_{1} \oplus V=M_{1} \oplus V^{\prime}$ where $V^{\prime}=\left(M_{1} \oplus V\right) \cap\left(M_{2} \oplus \cdots \oplus M_{n}\right)$. Since $V^{\prime}$ is a direct summand of $M_{2} \oplus \cdots \oplus M_{n}$, it is clear that $M_{1} \oplus V$ is a direct summand of $M$. It follows that $V$ is a direct summand of $M$. Now we consider the case $U=M \cap V \neq 0$. If $U=M_{1}$, then by modularity, we conclude that $V$ is a direct summand of $M$. If $U \neq M_{1}$, then $U$ is a minimal submodule of $M_{1}$. Let $S^{*}$ be the closure of $U$ in $V$. As $V$ is closed in $M, S^{*}$ must be closed in $M$ (see, for example, [2, Section $1.10(4)])$. Hence $l\left(S^{*}\right)$ is at least 2. Since $S^{*} \cap\left(M_{2} \oplus \cdots \oplus M_{n}\right)=0$, we have $S^{*} \oplus\left(M_{2} \oplus \cdots \oplus M_{n}\right)=M$. From here we conclude as before that $V$ is a direct summand of $M$. Thus $M$ is CS.

Conversely, assume that $t>1$. We use an idea in the proof of [4, Theorem 6] to show that $M$ is not CS. Suppose on the contrary that $M_{R}$ is CS. Then for $j=2, \ldots, t$, $M_{1} \oplus M_{j}$ is a CS module. Hence by [2, Section 7.3 (ii)], $M_{1}$ is $M_{j}$-injective. Let $S_{i}$ be the socle of $M_{i}$. Then $M_{1}$ is $S_{i}$-injective for any $i$.

Let $\varphi: S_{1} \rightarrow S_{j}$ be an isomorphism, and let $L=\left\{x+\varphi(x) \mid x \in S_{1}\right\}$. Then $L$ is a minimal submodule of $M_{1} \oplus M_{j}$. There are two possibilities:

(a) $L$ is closed in $M_{1} \oplus M_{j}$. Hence $L$ is a direct summand of $M_{1} \oplus M_{j}$. This is impossible by the Krull-Schmidt Theorem (compare with [1, Section 12.9]).

(b) $L$ is not closed in $M_{1} \oplus M_{j}$. Then the closure $L^{\prime}$ of $L$ in $M_{1} \oplus M_{j}$ has length at least 2. As $l\left(M_{1} \oplus M_{j}\right)=4$, we have $M_{1} \oplus M_{j}=L^{\prime} \oplus M_{j}=M_{1} \oplus L^{\prime}$. It follows $M_{1} \cong M_{j}$. Thus by [1, Section $\left.16.13(2)\right], M_{1}$ is $\left(M_{1} \oplus \cdots \oplus M_{t} \oplus M_{t+1} \oplus \cdots \oplus M_{n}=\right.$ $M)$-injective, a contradiction to the assumption that $l\left(E_{M}\left(M_{1}\right)\right)>2$.

The following example shows the existence of a ring $R(=B)$ of Theorem 1.1 with $A=C=0$, but $R$ is not right CS.

EXAMPLE 1 (compare with [4, Example 3.2]). Let

$$
R=\left(\begin{array}{lll}
\mathbb{C} & 0 & \mathbb{C} \\
0 & \mathbb{C} & \mathbb{C} \\
0 & 0 & \mathbb{C}
\end{array}\right)
$$

Then $R$ is a right (and left) SI ring, that is a ring over which every singular right (left) $R$-module is injective (see [3, Chapter 3]). Let

$$
e_{11}=\left(\begin{array}{lll}
1 & 0 & 0 \\
0 & 0 & 0 \\
0 & 0 & 0
\end{array}\right), \quad e_{22}=\left(\begin{array}{lll}
0 & 0 & 0 \\
0 & 1 & 0 \\
0 & 0 & 0
\end{array}\right), \quad e_{33}=\left(\begin{array}{lll}
0 & 0 & 0 \\
0 & 0 & 0 \\
0 & 0 & 1
\end{array}\right) \text {. }
$$


Then $R$ can be written in the form $R=e_{11} R \oplus e_{22} R \oplus e_{33} R$. It is clear that

$$
e_{33} R \cong \operatorname{Soc}\left(e_{11} R\right) \cong \operatorname{Soc}\left(e_{22} R\right), \quad l\left(e_{11} R\right)=l\left(e_{22} R\right)=2 .
$$

Moreover,

$$
E\left(e_{11} R\right)=\left(\begin{array}{lll}
\mathbb{C} & \mathbb{C} & \mathbb{C} \\
0 & 0 & 0 \\
0 & 0 & 0
\end{array}\right),
$$

and hence $l\left(E\left(e_{11} R\right)\right)=3$. Thus $R$ is a ring of Theorem 1.1 with $A=C=0$. However, by Lemma 3.1, $R$ is not right CS.

In light of Lemma 3.1, we can give some more information on the structure of the right ideal $B$ of $R$ in Theorem 1.1.

PROPOSITION 3.2. Let $R$ be a ring of Theorem 1.1 and $B$ be a right ideal of $R$ described in III (ii). Then $B_{R}=V_{1} \oplus \cdots \oplus V_{k} \oplus V_{k+1} \oplus \cdots \oplus V_{n}$, where each $V_{i}$ has a homogeneous socle such that for $i \neq j$, $\operatorname{Soc}\left(V_{i}\right) \not \operatorname{Soc}\left(V_{j}\right)$. Moreover, $V_{1}, \ldots, V_{k}$ are $C S$, and $V_{k+1}, \ldots, V_{n}$ are not $C S$.

Proof. As in Theorem 1.1 part III (ii), $B=B_{1} \oplus \cdots \oplus B_{m}$ where each $B_{i}$ is uniform of length 1 or 2 , and the injective hull of each simple submodule of $B_{R}$ has length 3. We can renumber the $B_{i}$ 's such that $B_{1}, \ldots, B_{k}$ has length 2 , each pair of these $B_{i}$ 's do not have isomorphic socles, and no $B_{j} \in\left\{B_{k+1}, \ldots, B_{m}\right\}$ is of length 2 and has a socle isomorphic to the socle of one of the $B_{i}$ 's for $i=1, \ldots, k$. The next $B_{k+1}, \ldots, B_{n}(n \leqslant m)$ have the property that each pair of them do not have isomorphic socles, each $B_{j}, k+1 \leqslant j \leqslant n$, has length 2 and for each of them there is at least one more $B_{t_{j}} \in\left\{B_{n}, \ldots, B_{m}\right\}$ such that $l\left(B_{t_{j}}\right)=2$ and $\operatorname{Soc}\left(B_{t_{j}}\right) \cong \operatorname{Soc}\left(B_{j}\right)$. The socle of each $B_{t} \in\left\{B_{n+1}, \ldots, B_{m}\right\}$ is isomorphic to either the socle of some $B_{i}, 1 \leqslant i \leqslant k$, or the socle of some $B_{j}$ with $k+1 \leqslant j \leqslant n$.

Now let $\left[B_{i}\right]$ be the direct sum of all $B_{i^{\prime}} \in\left\{B_{1}, \ldots, B_{m}\right\}$ with $\operatorname{Soc}\left(B_{i^{\prime}}\right) \cong \operatorname{Soc}\left(B_{i}\right)$. By the structure of the right ideals $A, B, C$ of $R$ in Theorem 1.1, there is no nonzero homomorphism of any submodule of $A_{R}$ and respectively, of any submodule of $C_{R}$ to $B_{R}$. This implies that every submodule of $B$ is $A$ - and $C$-injective. Hence any $B$-injective submodule of $B$ is injective. Thus we can apply Lemma 3.1 to see that $\left[B_{1}\right], \ldots,\left[B_{k}\right]$ are CS modules, and $\left[B_{k+1}\right], \ldots,\left[B_{n}\right]$ are not CS, proving Proposition 3.2.

\section{Acknowledgements}

The authors wish to express their thanks to the referee for many useful suggestions and comments. 


\section{References}

[1] F. W. Anderson and K. R. Fuller, Rings and categories of modules, GTM 13, 2nd edition (Springer, New York, 1992).

[2] N. V. Dung, D. V. Huynh, P. F. Smith and R. Wisbauer, Extending modules (Pitman, London, 1994).

[3] K. R. Goodearl, Singular torsion and the splitting properties, Mem. Amer. Math. Soc. 124 (Amer. Math. Soc., Providence, RI, 1972).

[4] D. V. Huynh, 'Structure of some noetherian SI rings', J. Algebra 254 (2002), 362-374.

[5] D. V. Huynh and S. T. Rizvi, 'On some classes of artinian rings', J. Algebra 223 (2000), 133-153.

[6] G. Ivanov, 'Non-local rings whose ideals are quasi-injective', Bull. Austral. Math. Soc. 6 (1972), 45-52.

[7] T. Y. Lam, Lectures on modules and rings, GTM 189 (Springer, New York, 1999).

[8] R. Wisbauer, Foundations of module and ring theory (Gordon and Breach, Reading, 1991).

Department of Mathematics

Ohio University

Athens

Ohio 45701

USA

e-mail: huynh@math.ohiou.edu
Department of Mathematics

The Ohio State University at Lima

Lima

Ohio 45804

USA

e-mail: rizvi.1@osu.edu 